\title{
Application of a novel magnetic anchoring and traction technique in thoracoscopic esophagectomy: a swine experiment
}

\author{
Wenwen Chen ${ }^{1,2 \#}$, Yunhao Li $^{1 \#}$, Rongkai Qian ${ }^{1,2}$, Meiling Jiang ${ }^{1,2}$, Lu Lv ${ }^{1,2}$, Siyu Ren ${ }^{1,2}$, Aihua Shi ${ }^{3}$, \\ Xiaopeng Yan ${ }^{3,4}$, Junke Fu ${ }^{1}$, Haohua Wang ${ }^{3}$, Feng $\mathrm{Ma}^{3}$, Yi $\mathrm{Lv}^{3,4}$, Yong Zhang ${ }^{1,3}$ \\ ${ }^{1}$ Department of Thoracic Surgery, the First Affiliated Hospital of Xi'an Jiaotong University, Xi'an, China; ${ }^{2}$ Xi'an Jiaotong University Health Science \\ Center, Xi'an, China; ${ }^{3}$ National Local Joint Engineering Research Center for Precision Surgery \& Regenerative Medicine, the First Affiliated \\ Hospital of Xi'an Jiaotong University, Xi'an, China; ${ }^{4}$ Department of Hepatobiliary Surgery, the First Affiliated Hospital of Xi'an Jiaotong University, \\ Xi'an, China \\ Contributions: (I) Conception and design: X Yan, Y Lv, Y Zhang; (II) Administrative support: A Shi, Y Lv, Y Zhang; (III) Provision of study materials \\ or patients: A Shi, X Yan, J Fu, H Wang, F Ma; (IV) Collection and assembly of data: All authors; (V) Data analysis and interpretation: All authors; (VI) \\ Manuscript writing: All authors; (VII) Final approval of manuscript: All authors. \\ \#These authors contributed equally to this work. \\ Correspondence to: Yong Zhang. Department of Thoracic Surgery, the First Affiliated Hospital of Xi'an Jiaotong University, Xi'an 710061, China. \\ Email: zhangyongyang05@163.com; Yi Lv. Department of Hepatobiliary Surgery, the First Affiliated Hospital of Xi'an Jiaotong University, Xi'an \\ 710061, China. Email: luyi169@126.com.
}

Background: Effective traction and dissection of the esophagus are key steps during thoracoscopic esophagectomy. In traditional methods, a separate trocar for the traction instruments or thoracic punctures are adopted to externally retract the esophageal loop. However, both methods bring about chest wall damage that is associated with increased morbidity and mortality. The magnetic anchoring and traction system can not only achieve exposure and pulling multi-directional flexible but also reduce the number of transthoracic ports and trocars used, and then avoid the chopstick effect in surgery. We aimed to verify the feasibility and safety of a self-designed magnetic anchoring and traction system in assisted thoracoscopic esophagectomy.

Methods: Ten healthy pigs were used as the experimental objects. A magnetic anchoring and traction system composed of an external unit and internal unit was designed, then the requirements and stress characteristics of esophageal pulling and exposure during thoracoscopic esophagectomy were analyzed. The internal unit was introduced through the 5 th intercostal space port and was secured to the right wall of the esophagus, the external unit was placed on the surface of the chest wall to allow pairing with the internal unit. The external unit was moved on the chest wall to help exposing operative field.

Results: Ten pigs underwent a 3-port thoracoscopic esophagectomy using a magnetic anchoring and traction technique, and all operations were successful. The system provided adequate traction force to pull the esophagus. The external unit could move freely outside the chest wall, enabling suitable positioning of the esophagus for dissection.

Conclusions: The novel magnetic anchoring and traction system in thoracoscopic esophagectomy is safe and feasible, and has the potential for clinical application.

Keywords: Thoracoscopy; thoracoscopic esophagectomy; magnetic anchoring and traction system; porcine model

Submitted Sep 06, 2021. Accepted for publication Nov 09, 2021.

doi: $10.21037 /$ atm-21-5359

View this article at: https://dx.doi.org/10.21037/atm-21-5359 


\section{Introduction}

Thoracoscopic esophagectomy has resulted in improvements in recovery time, postoperative pain, and cosmesis. However, the prevailing available technology still requires the placement of multiple ports through the chest wall. Each of these transthoracic punctures is associated with risks of morbidity and mortality (1), prompting surgeons to develop less invasive techniques to reduce the number of transthoracic ports and trocars used.

Minimally invasive esophagectomy (MIE) is now widely accepted as the procedure of choice for early-stage esophageal cancer. Recently, single-port thoracoscopic esophagectomy has been reported (2). However, minimizing the invasiveness of this surgery has its own challenges. The insertion of multiple instruments through a single incision can lead to instrument "collision" as they all compete in the same trajectory toward the area or organ of interest. These technical challenges hinder single-port minimally invasive surgeries from entering mainstream surgical practice (3).

To facilitate thoracoscopic surgery, we intended to develop and optimize a technique enabling the entry of required instruments into the area under investigation without them having to "take up" and compete for port site space. Compared with traditional thoracoscopic esophagectomy, it reduces damage to the chest wall, avoids the chopstick effect, and provides multidirectional pulling and exposure. To this end, the magnetic anchoring and traction system (MATS), a system that consists of 2 magnetic elements (4) paired through the thoracic wall, has shown promise. This technique is called the magnetic anchoring and traction technique (MATT) that uses magnetic materials to generate force through indirect contact to achieve traction and suspension of the external unit to the internal unit. We aimed to design and evaluate the usefulness of a novel MATS for the traction and dissection of the esophagus during thoracoscopic esophagectomy.

We present the following article in accordance with the ARRIVE reporting checklist (available at https://dx.doi. org/10.21037/atm-21-5359).

\section{Methods}

Ten pigs, including 6 female and 4 male pigs (weight: $23.1-$ $30 \mathrm{~kg}$, age: 2-3 months), were housed in separate cages, fed a standard diet, and allowed free movement during the experimental period. All pigs were purchased from the
Laboratory Animal Research Center of the Xi'an Jiaotong University. Experiments were performed under a project license (No. XJTULAC2019-1270) granted by ethics board of Xi'an Jiaotong University, in compliance with the standard ethical guidelines under the control of the ethics board mentioned above. A protocol was prepared before the study without registration.

In this study, the MATS consisted of an external unit (an external handheld magnet) and an internal unit [a stainless steel cylinder (SSC) connected to a tissue gripper]. The external magnet is cylindrical, composed of neodymiumiron-boron (NdFeB, N45), and covered with a nickel alloy. The outer diameter and height of the external magnet are 50 and $75 \mathrm{~mm}$, respectively. This magnet is wrapped in a handheld device made of acrylonitrile butadiene styrene plastic (Figure 1A). The outer diameter and height of the internal SSC are 11 and $10 \mathrm{~mm}$, respectively (Figure $1 B$ ). The length and jaw width of the tissue gripper are 26 and $8 \mathrm{~mm}$, respectively (Figure 1C). The weight of the internal unit is $11.7 \mathrm{~g}$ (tissue gripper $4.2 \mathrm{~g}$ and SSC $7.5 \mathrm{~g}$ ). The gripper applier (Hangzhou Kangji Medical Instrument Co., Ltd., Hangzhou, Zhejiang, China) (Figure 1D) is used to put the internal unit into the body. The magnetic field of the external magnet is 3,500 GS. The attraction between the external magnet and the internal SSC at zero distance is $35 \mathrm{~N}$, decreasing exponentially with distance (Figure 2).

To determine the range of chest wall thickness and the force required to pull the thoracic part of the esophagus, we measured the chest wall thickness at the $6^{\text {th }}$ intercostal space (ICS), the inferior angle of the scapular line, and the posterior axillary and anterior axillary lines in 10 pigs (Figure 3). Then, the middle part of the thoracic esophagus was mobilized and encircled with the esophagus string loop. The traction force required to gently pull the esophagus to the maximum extent was detected with a tension meter. We defined the "maximum extent" as the state in which the esophagus is completely pulled and there is outer membrane tear or bleeding.

The animals were administered general anesthesia using an intramuscular injection of $0.08 \mathrm{~mL} / \mathrm{kg}$ of Luminal (phenobarbital sodium), intubated, and placed in the left lateral decubitus position. A 1-cm incision was made for the endoscope in the $7^{\text {th }}$ ICS at the midaxillary line. Two additional 1 -cm incisions were made as the main working ports in the $3^{\text {rd }}$ and $5^{\text {th }}$ ICS at the anterior axillary line (Figure 4).

We used a monopolar electrocautery hook to dissect the mediastinal pleura and soft tissue from the esophagus at the level of the $7^{\text {th }}$ thoracic vertebrae. The internal unit was 


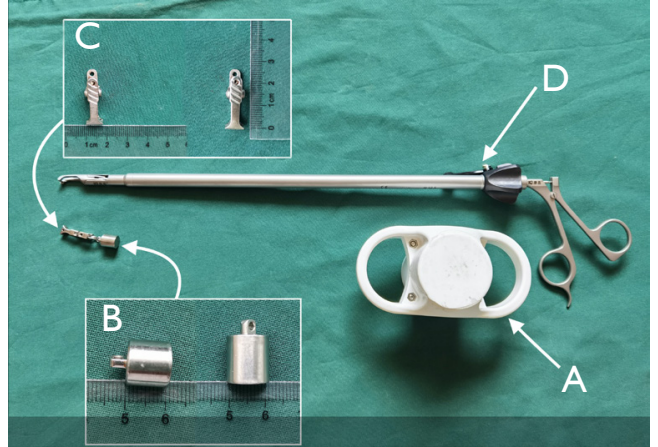

Figure 1 Magnetic anchoring and traction system. (A) External handheld magnet. (B) Internal stainless steel cylinder. (C) Tissue gripper. (D) Gripper applier (Hangzhou Kangji Medical Instrument Co., Ltd.).

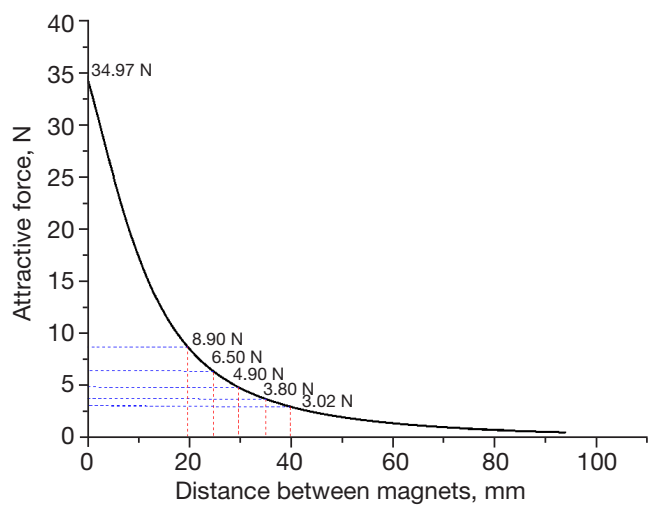

Figure 2 Magnetic attraction force curve. The magnetic attraction force decreases exponentially with the increase in distance.

introduced through the $5^{\text {th }}$ ICS port. The tissue gripper was secured to the right wall of the esophagus using a gripper applier. The external magnet was placed on the surface of the chest wall to allow pairing with the internal SSC. The MATS exerted a traction force on the esophagus, which could then be drawn to the specific position required for dissection. The external magnet, which was able to move freely on the chest wall, helped obtain an optimal operative field (Figure 5). The MATS enabled easy dissection of the esophagus. After the process was completed, the internal SSC was uncoupled from the external magnet, the tissue gripper was released from the esophagus, and the internal unit was removed through the $5^{\text {th }}$ ICS port. All incisions were closed. All the animals were euthanized by the administration of a lethal dose $(1-2 \mathrm{mmol} / \mathrm{kg})$ of potassium chloride.

\section{Statistical analysis}

Quantitative variables, such as weight, Chest wall thickness (CWT), and attraction forces, each data were measured 3 times and were summarized as means and standard deviations. All statistical analyses were performed with IBM SPSS Statistics version 23.0 software (IBM Corp., Armonk, NY, USA).

\section{Results}

The chest wall thicknesses of the 10 animals and the force needed for esophageal traction are reported in Table 1.

All operations were uneventful and were performed using a minimally invasive route ( 3 ports). The mean operation time was $30 \mathrm{~min}$ and the mean intraoperative blood loss was $10 \mathrm{~mL}$. The force of attraction between the external magnet and the internal SSC was adequate to ensure sufficient traction of the esophagus to expose the operative field (Figure 5B). The traditional grasper used for esophageal traction was not needed, and only 2 instruments were required to dissect the esophagus through the working ports. Meanwhile, the number of transthoracic punctures and the number of trocars required were reduced.

\section{Discussion}

When using traditional methods in thoracoscopic-assisted esophagectomy, the thoracoscopic tissue grasper is inserted through the port on the chest wall to draw the esophagus and align it on a suitable anatomical plane. In our study, a MATS was used instead of a thoracoscopic tissue grasper for esophageal traction. The internal unit was inserted into the chest cavity through a thoracic port and then controlled by pairing it with an externally held magnet. The thoracic port was kept open for the insertion of other working instruments after the insertion of the internal unit.

The use of the MATS can allow positioning of the esophagus that does not require the insertion of a separate transthoracic trocar. This technology has been used in various surgical fields. In a prospective, multicenter clinical trial, Rivas et al. evaluated a MATS during a reducedport laparoscopic cholecystectomy. The exposure of the surgical site was rated as "excellent" by $90 \%$ of the surgeons, and $10 \%$ rated it as "sufficient" (5). Padilla et al. argued that the MATS can provide excellent triangulation and improve ergonomics, and that a magnetassisted single trocar appendectomy procedure can be 

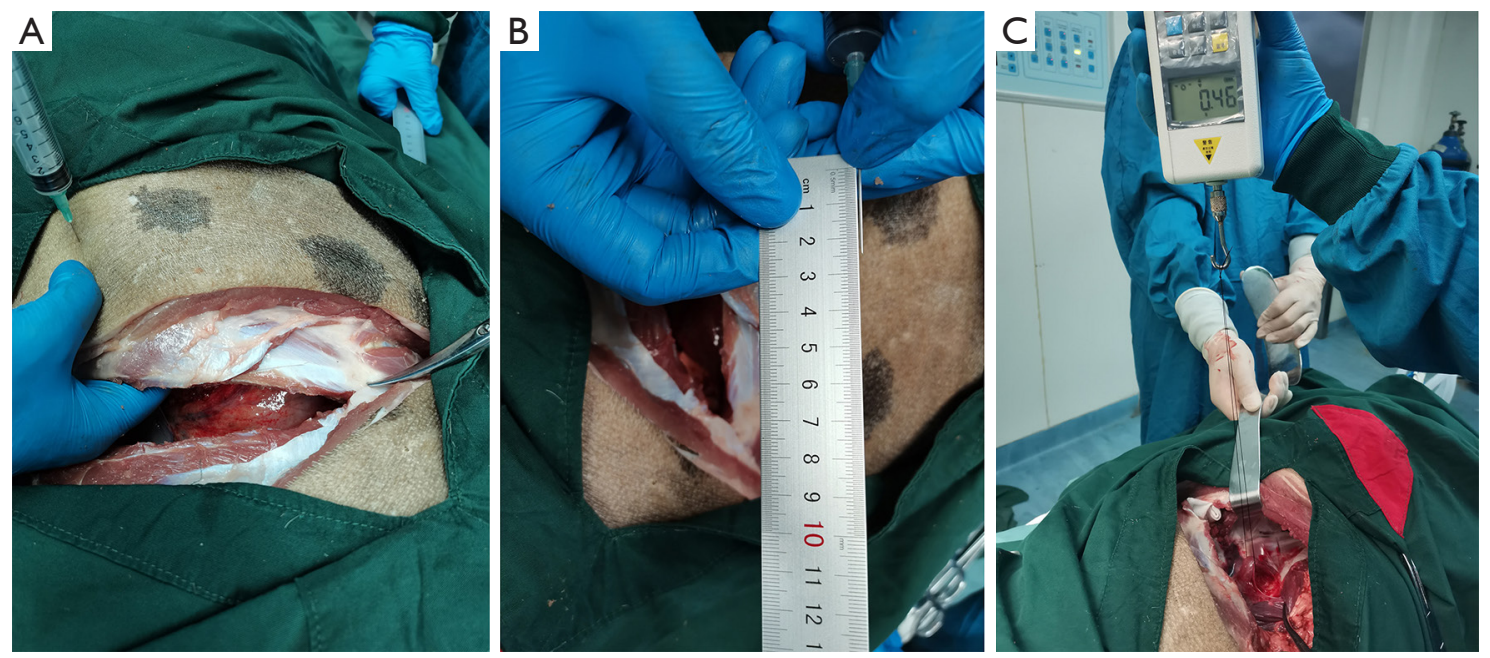

Figure 3 Measurement of the chest wall thickness and the pulling force required for esophageal traction. (A) Use of a 10-mL syringe to determine the chest wall thickness. (B) Measurement of the syringe tip length. (C) Measurement of the pulling force for traction and dissection of the esophagus.

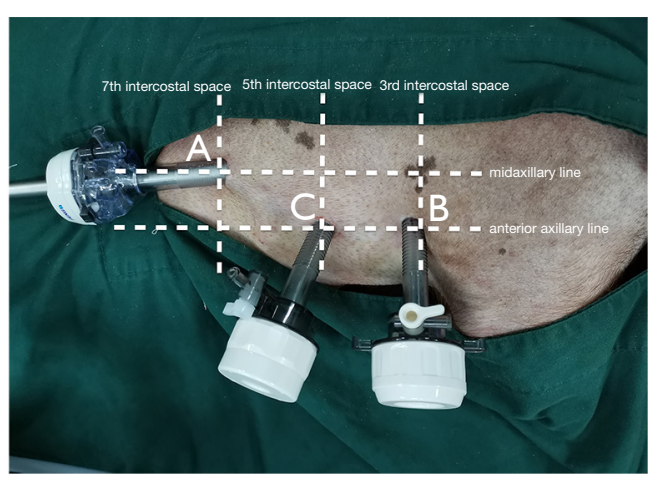

Figure 4 Surgical incisions. (A) A 1-cm incision for the endoscope in the 7 th intercostal space (ICS) at the midaxillary line. (B,C) A $1-\mathrm{cm}$ incision in the $3 \mathrm{rd}$ and 5 th ICS at the anterior axillary line.

performed without the aid of a surgical assistant (6). The MATS was also shown to be safe and effective in Nissen fundoplication, gastrojejunostomy, splenectomy, ovarian tumor/cyst resection, retroperitoneal lymphangioma resection, nephrectomy, prostatectomy, adrenalectomy, total abdominal colectomy, and pulmonary wedge resection (7-9). Moreover, it was shown to overcome the collisions among working instruments, build operative triangulation, and reduce transabdominal ports in laparoscopic surgery (10). However, there is a lack of reports on thoracoscopic esophagectomy with respect to the application of the MATT.
We performed this experiment on pigs to test the feasibility and effectiveness of a novel MATS in MIE. We used the system in a 3-port thoracoscopic esophagectomy. The attraction of the external magnet to the internal SSC was sufficient for exposure of an adequate surgical field. The external magnet was able to easily control the internal unit's movement. The tension needed for esophageal dissection was provided by the internal tissue gripper that was guided by the movement of the external magnet. There was sufficient surgical vision for the operation. The number of instruments used in esophageal traction, the number of transthoracic punctures, and the trocars required were also reduced.

In this experiment, we measured the pulling force required for the traction of the esophagus in 10 pigs. Our results showed that it took only $0.2 \mathrm{~N}$ to move the esophagus gently, $0.4-2.0 \mathrm{~N}$ to dissect the esophagus, and $2.2 \mathrm{~N}$ or more to pull the esophagus to the maximum extent. The mean thickness of the chest wall at the inferior angle of the scapular line for the 10 pigs was $2.5 \mathrm{~cm}$, which was the thickest among the 3 lines (Table 1). The attraction force between the external magnet and internal SSC was $3.02 \mathrm{~N}$ at $4 \mathrm{~cm}$ (Figure 2). Excluding 0.12 and $0.24 \mathrm{~N}$ required to resist the gravity of the internal units, these forces fully met the pulling force requirement $(0.3-2.2 \mathrm{~N})$ for dissecting the esophagus.

Our study has several limitations. First, the sample size was not sufficient to generalize our results. Second, the 

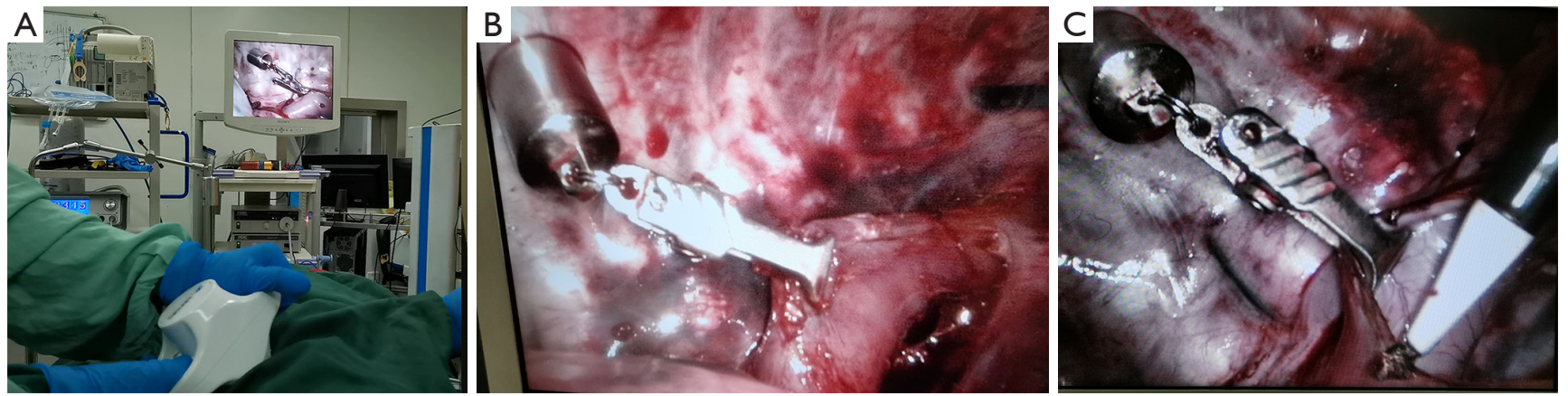

Figure 5 An optimal operative field was obtained by moving the external magnet. (A) The external magnet paired with the internal stainless steel cylinder to obtain an optimal operative field. (B) Retraction of the esophagus by using the magnetic anchoring and traction system. (C) Use of electrocautery to dissect the esophagus.

Table 1 Results of chest wall thicknesses and force needed for esophageal traction

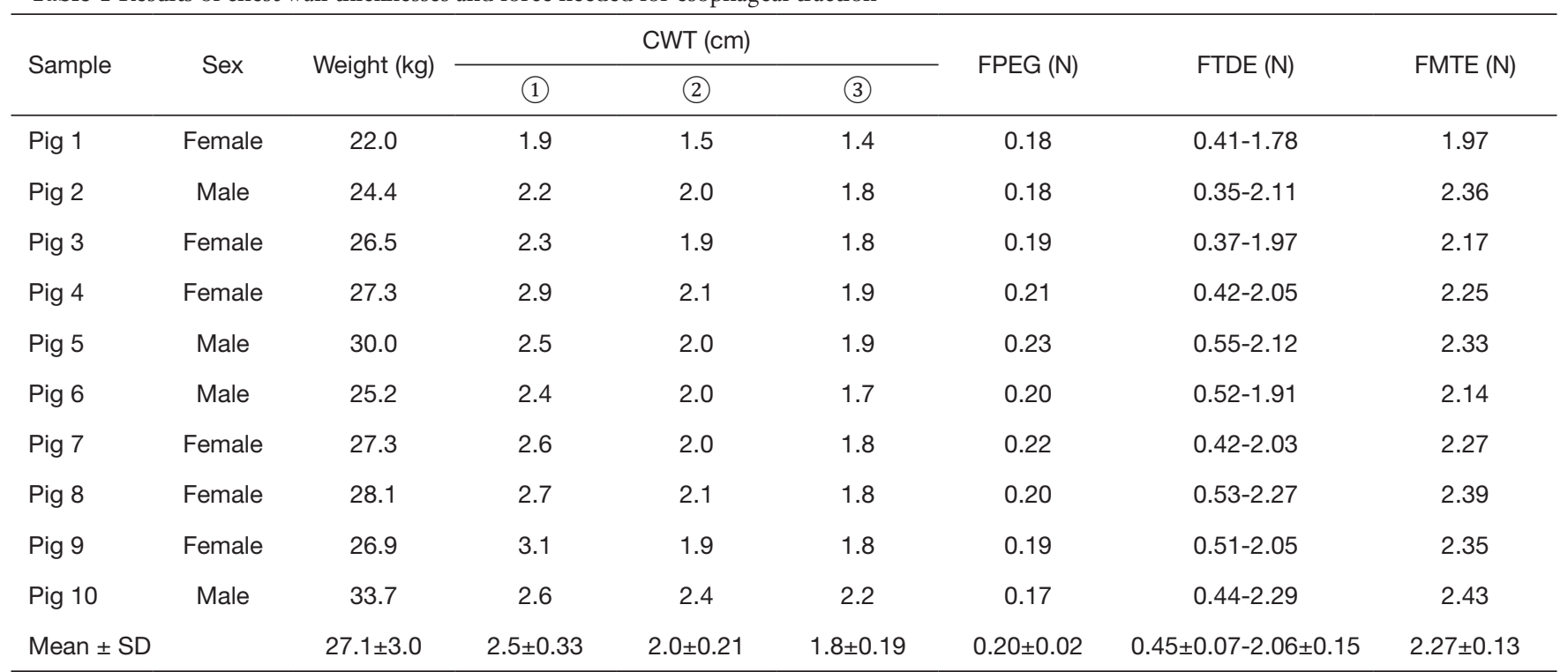

M \pm SD: mean standard deviation; CWT, chest wall thickness: (1) at the inferior angle of scapular line, (2) at the posterior axillary line, (3) at the anterior axillary line; FPEG, force for pulling esophagus gently; FTDE, force for traction and dissection of esophagus; FMTE, force for maximal traction of esophagus.

shape of the thoracic cavity is irregular, and the magnetic attraction force was insufficient or too powerful in some positions, this will require further improvement of the MATS. Third, some surgical instruments will be affected by magnetic force. Therefore, non-magnetic instruments need to be developed for the widespread application of this technique in clinical practice. In the future, we have even more bold ideas, combine with artificial intelligence to develop intelligent magnetic anchoring traction equipment, for example, eye tracking system which automatically adjusts the endoscope and the magnetic force between the internal and external units to avoid tissue torn or bleeding.

In conclusion, our study using the MATS demonstrates a novel technique of esophageal traction that reduces the need for extra transthoracic ports. The force required for esophageal traction in this study was not weaker than that required for traction by the instruments used in current methods. Thus, it further verifies the feasibility of the MATS for thoracoscopic esophagectomy and its clinical application potential in the future. 


\section{Acknowledgments}

We thank the Taylor \& Francis Editing Services Team for their language edits.

Funding: This work was supported by the Fundamental Research Funds for the Central University of China (No. xzd012020026), the Funds for Key Research and Development Program Projects in Shaanxi Province, China (No. 2019SF-014), and Bethune Charitable Foundation (No. HZB-20181119-070).

\section{Footnote}

Reporting Checklist: The authors have completed the ARRIVE reporting checklist. Available at https://dx.doi. org/10.21037/atm-21-5359

Data Sharing Statement: Available at https://dx.doi. org/10.21037/atm-21-5359

Conflicts of Interest: All authors have completed the ICMJE uniform disclosure form (available at https://dx.doi. org/10.21037/atm-21-5359). The authors report that this work was supported by the Fundamental Research Funds for the Central University of China (No. xzd012020026), the Funds for Key Research and Development Program Projects in Shaanxi Province, China (No. 2019SF-014), and Bethune Charitable Foundation (No. HZB-20181119-070). The authors have no other conflicts of interest to declare.

Ethical Statement: The authors are accountable for all aspects of the work in ensuring that questions related to the accuracy or integrity of any part of the work are appropriately investigated and resolved. Experiments were performed under a project license (No. XJTULAC2019-1270) granted by ethics board of Xi'an Jiaotong University, in compliance with the standard ethical guidelines under the control of the ethics board mentioned above. A protocol was prepared before the study without registration.

Open Access Statement: This is an Open Access article distributed in accordance with the Creative Commons Attribution-NonCommercial-NoDerivs 4.0 International License (CC BY-NC-ND 4.0), which permits the noncommercial replication and distribution of the article with the strict proviso that no changes or edits are made and the original work is properly cited (including links to both the formal publication through the relevant DOI and the license).
See: https://creativecommons.org/licenses/by-nc-nd/4.0/.

\section{References}

1. Tamura M, Shimizu Y, Hashizume Y. Pain following thoracoscopic surgery: retrospective analysis between single-incision and three-port video-assisted thoracoscopic surgery. J Cardiothorac Surg 2013;8:153.

2. Hu W, Yuan Y, Chen L. Single-Port Thoracoscopic Minimally Invasive Esophagectomy for Esophageal Cancer. World J Surg 2019;43:567-70.

3. Martin-Ucar AE, Aragon J, Bolufer Nadal S, et al. The influence of prior multiport experience on the learning curve for single-port thoracoscopic lobectomy: a multicentre comparative study†. Eur J Cardiothorac Surg 2017;51:1183-7.

4. Lv Y, Shi Y; Scientific Committee of the First International Conference of Magnetic Surgery* Xi'an consensus on magnetic surgery. Hepatobiliary Surg Nutr 2019;8:177-8.

5. Rivas H, Robles I, Riquelme F, et al. Magnetic Surgery: Results From First Prospective Clinical Trial in 50 Patients. Ann Surg 2018;267:88-93.

6. Padilla BE, Dominguez G, Millan C, et al. Initial experience with magnet-assisted single trocar appendectomy in children. J Laparoendosc Adv Surg Tech A 2013;23:463-6.

7. Steinberg RL, Johnson BA, Cadeddu JA. Magnetic-assisted robotic surgery: initial case series of reduced-port robotic prostatectomy. J Robot Surg 2019;13:599-603.

8. Yan X, Li Y, Fu J, et al. Magnetic anchoring technique assisted thoracoscopic pulmonary wedge resection 3 cases (in Chinese). Chin J of Clin Thorac and Cardiovasc Surg 2020;27:229-30.

9. Padilla BE, Dominguez G, Millan C, et al. The use of magnets with single-site umbilical laparoscopic surgery. Semin Pediatr Surg 2011;20:224-31.

10. Best SL, Cadeddu JA. Use of magnetic anchoring and guidance systems to facilitate single trocar laparoscopy. Curr Urol Rep 2010;11:29-32.

(English Language Editor: C. Betlzar)

Cite this article as: Chen W, Li Y, Qian R, Jiang M, Lv L, Ren S, Shi A, Yan X, Fu J, Wang H, Ma F, Lv Y, Zhang Y. Application of a novel magnetic anchoring and traction technique in thoracoscopic esophagectomy: a swine experiment. Ann Transl Med 2021;9(22):1663. doi: 10.21037/ atm-21-5359 